\title{
Improvements to the Registry for Stroke Care Quality (RES-Q) based on first year user feedback
}

\author{
Andreea $\mathrm{Grecu}^{1}$, Steven Simsic ${ }^{1}$, Marie Jankujova ${ }^{1}$, Robert Mikulik ${ }^{1,2}$
}

\author{
1 International Clinical Research Center, St. Anne's University Hospital Brno, Czech Republic; 2 First Department of Neurology, St. Anne's University and Faculty of Medicine, Masaryk University, Brno, Czech Republic
}

\section{INTRODUCTION}

The Registry of Stroke Care Quality (RES-Q) was initially launched in November of 2016 as a tool for monitoring, analysing, and improving stroke care processes in hospitals throughout Europe. RES-Q was developed as a part of the European Stroke Organization's Enhancing and Accelerating Stroke Treatment (ESO-EAST) Programme with the goal of ensuring that quality stroke treatment is delivered effectively, and equally throughout Europe.

RES-Q was initially launched in approximately 150 hospitals in 22 ESO-EAST countries, primarily located in Eastern Europe and Central Asia. In 18 months since the launch of the project, RES-Q has grown to include the participation of over 450 hospitals in more than 40 countries worldwide. This rapid growth required that we analyse the needs of all collaborating countries, professional societies, and hospitals to determine the most effective ways to evaluate their needs in understanding how stroke care is delivered to their patients. Based on effective ways to evaluate their needs in understanding how stroke care is delivered to their patients. Based
this analysis, we have implemented multiple changes, both strategic, and technical to the RES-Q platform.

The RES- $Q$ technical platform is centred around a web-based questionnaire, built on an open-source platform for clinical trial management. Optimizing the platform to meet the evolving demands of RES-Q collaborators required that analysis of needs and implementation of changes needed to be concurrent with the ongoing operation of the registry. As such, proposed changes needed to be evaluated in terms of their necessity, development requirements, and ability to integrate with existing functionality.

RES-Q Patient Enrollment April 2018

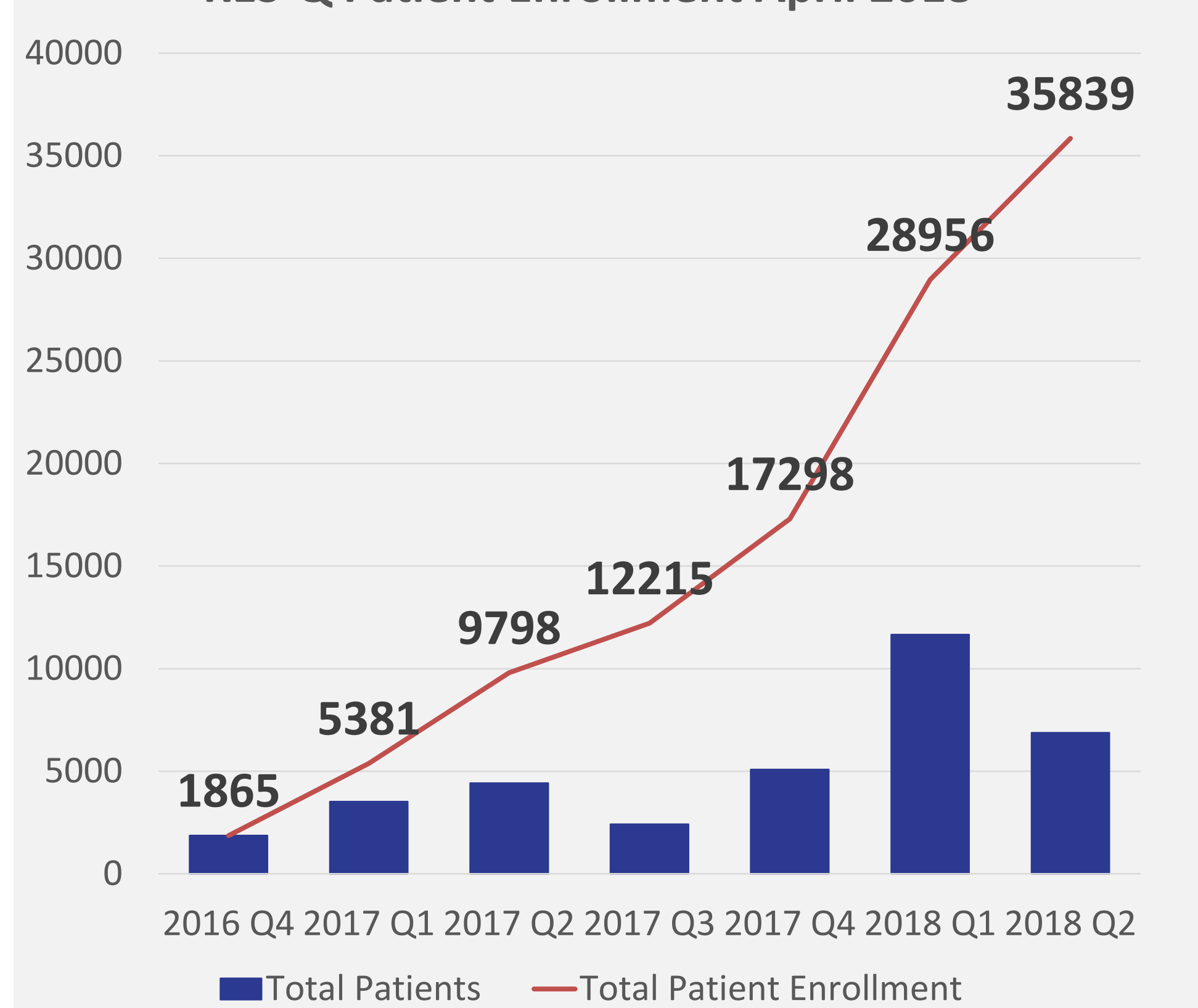

RES-Q Hospital Enrollment April 2018

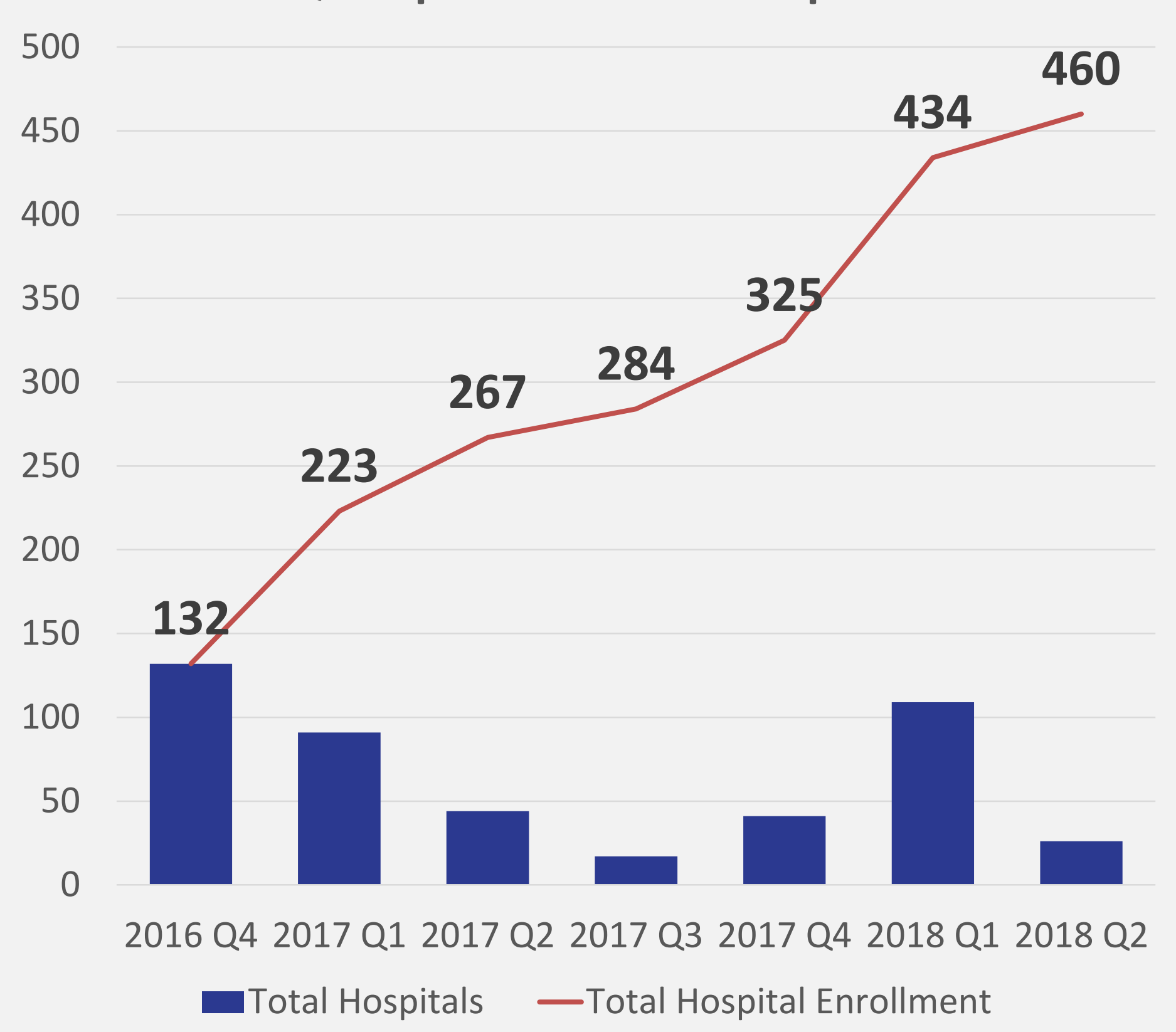

\section{IMPLEMENTATION}

\section{Translations}

The need to implement translations in RES-Q was expected, and so a framework for implementing this already existed. Translations are not an integrated part of the open source platform used by RES-Q, however the implementation was relatively straightforward. The base questionnaire implements a mapping from question and response text to numerical values. This mapping allows us to substitute any text for the base questionnaire text without needing to make changes to the database directly. Each translation exists as a version of the questionnaire form on the platform, so participating hospitals can access and use multiple translations (e.g. an English version, and a Czech version) and still have all data collected at their hospital.

\section{Revised Questionnaire}

Implementing the revised questionnaire was the most complex process in the RES-Q improvement plan. After processing the initial feedback, multiple meetings were held between participating hospitals, National Coordinators, National Professional Society members, and RES-Q development team members to establish a new questionnaire.

Once an initial revised form was approved, a test version was implemented using a similar versioning system as discussed in the translation implementation section. However, as this revised version contained new variables, instead of being created as a new version of an existing form, it was created as a new form entirely. Structurally, this means that within the registry platform, if a user had access to the original questionnaire, and the new questionnaire, they would be able to see both at the same time, and would be able to enter data into both.

This revised questionnaire was tested by participating sites and other stakeholders in several rounds of iterative development. The resulting questionnaire became RES-QV2.0 and was launched for use in the second officia international data collection period in March 2018. As the development took longer than anticipated, RES-Qv2.0 was not mandatory, and we kept the functionality allowing hospitals to enter data in RES-Qv1.0 or RES-Qv2.0. This resulted in more burdensome data processing afterwards, however this was deemed necessary to prevent hospitals from being unable to fill in data due to lacking training or a translation of the RES-Qv2.0 questionnaire. Based on feedback obtained during 2017, the questionnaire was revised to include 3 new metrics, 4 new patient pathways, and multiple new response options to existing questions. Implementation of most new metrics involved development of new branching logic for the dynamic web form.

\section{Improved Reporting}

While most stakeholders indicated that the content of the existing RES-Q reports was useful and informative, an implementation for more frequent reporting was required. Initial reports from the first international data collection period were largely processed manually. The registry platform is capable of exporting data in multiple formats automatically, including XLSX, CSV, XML, SPSS, and CDISC ODM. However, generating descriptive statistics, graphs, and presentations was a time consuming process. An additional requirement was that reports needed to be generated for multiple stakeholders as hospitals were using RES-Q to participate in multiple ongoing projects.

To improve this functionality we automated much of the process using custom developed software to allow us to generate reports to meet each stakeholder's requirements. This customization allows RES-Q to produce reports for National level summaries, Hospital level reporting, as well as conference and paper publication, and even participation in the ESO Angels Awards.

\section{Custom Site Partitioning}

As of April 2018 this implementation is still in a test phase and is currently only utilized in 2 countries which indicated that it would be mandatory for using RES-Q in their centres. This is a structurally difficult change to implement within the RES-Q platform as hospitals are identified within the registry by an ID which is associated with their country. This was a decision made during initial platform development based on the presumed use case where National Professional Societies would be the primary users of RES-Q.

To avoid re-implementing the registry platform to accommodate a multi-tiered structure (e.g. National $>$ Regional > Hospital) we chose to implement this nominally in the platform, but primarily in the data processing phase. Currently, hospitals can only access their own data, regardless of their national affiliation. We used this permission restriction to allow us to extend the conventional site ID to include a regional identifier as well. This is not meaningful in the permission structure of the platform, as it does not need to be. It does however allow us to identify data as belonging to either a national, or regional group during processing.

\section{dentifying Needs}

The initial component of our improvement plan for upgraded registry functionality was an evaluation of potential stakeholders. We identified 5 relevant stakeholder groups which we would need to consult regarding necessary features, and the effectiveness of our implementation: 1. National Coordinators / Professional Societies, 2. Clinicians, 3. Local Coordinators / Registry Users, 4. Partner Organizations, 5. Data Managers / Developers.

With the list of proposed stakeholders in mind, we then developed a list of proposed questions / subjects based ne initial data collection which would be used to gather feedback and guide the direction of future development, targeted at gathering negative feedback.

What do we need to measure? This relates not only to the metrics themselves in terms of the data collected, but also how we design the data analysis algorithms to process the data.

What metrics are currently missing? We wanted to identify which areas in the questionnaire left users without an appropriate response option.

What doesn't work, or is hard to use? This was focused on the functionality of the user interface, and how interaction with the site felt.

What barriers to utilization / adoption exist in hospitals and countries? This is a broad question, and Evaluating Feedback

Feedback was collected following the first international data collection in March 2017, and being continuously collected thereafter. Beginning in September 2017, we began collating submitted feedback and preparing a new development strategy. From the feedback collected, 4 specific needs were identified as priorities for immediate development work.

Translations. Many countries identified this as a barrier to hospital enrolment as well as to accurate and forly data entry. A total site translation for every country was not seen as necessary, however translations or the questionnaire were a priority.

Revised questionnaire. Based on both feedback from clinicians, as well as from analysis of the data, we determined that the questionnaire would need to be updated. Clinicians identified multiple areas where the existing response options were inadequate, as well as additional information that should be included. Analysis of the data itself revealed questions where known values deviated significantly from the data entered suggesting that the question was unclear.

Improved reporting. RES-Q was initially developed to handle annual data collection over a one month period. During 2017 many countries and hospitals continued to use RES-Q for ongoing quality monitoring outside of the annual data collection period. As RES-Q is also an official registry for the ESO Angels Awards, this is expected to continue. It was decided that more frequent (quarterly) reporting would be required.

Custom hospital partitioning. The initial design of RES-Q focused on a project design whereby results were communicated to a designated national coordinator which was responsible for managing RES-Q within their country. Some countries identified this as a barrier to use as their healthcare system would not accept national data sharing, so a new provincial/regional schema would need to be implemented.

RES-Q Collected Variables

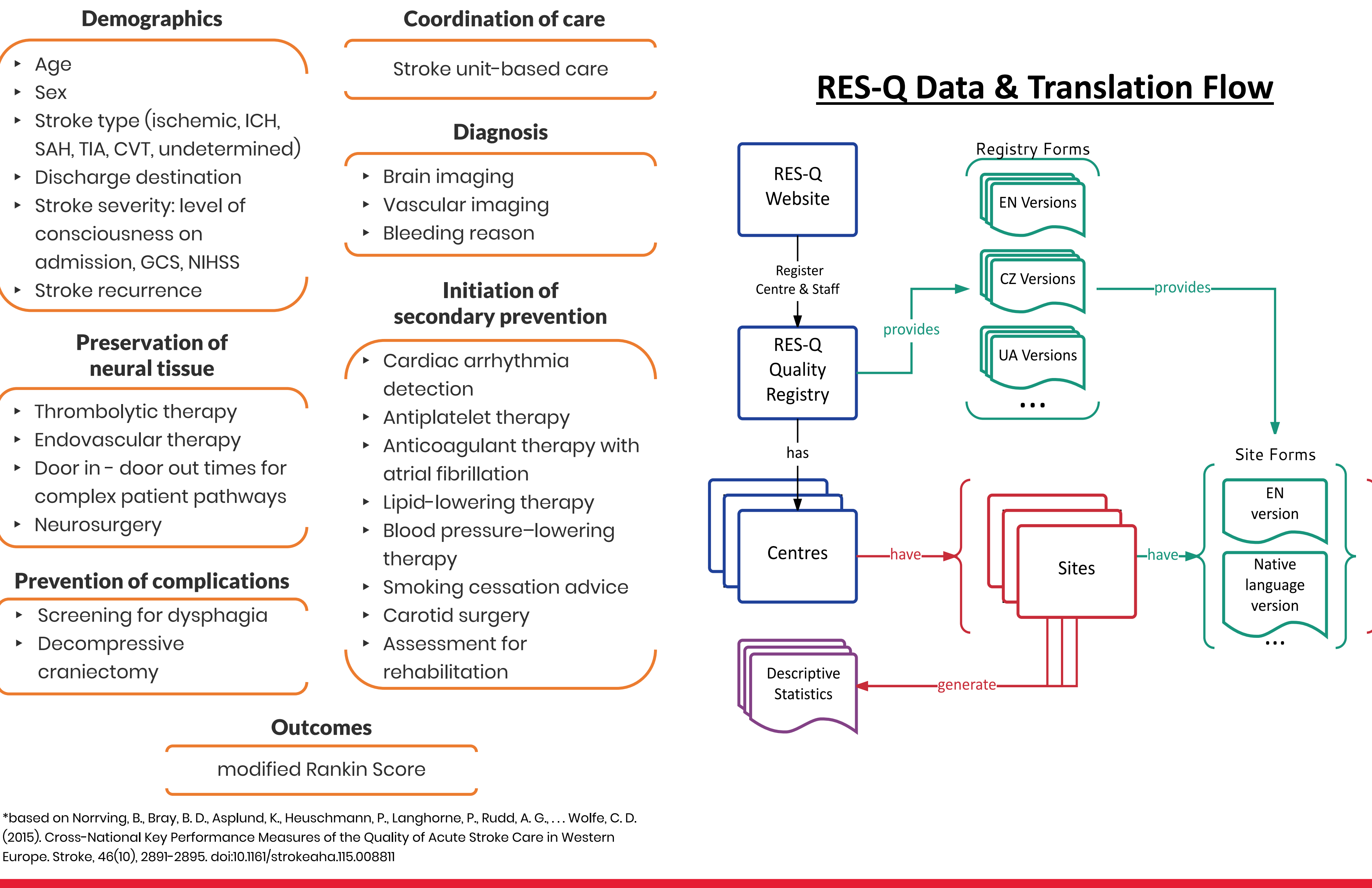

Improved Reporting

Data reporting is the most requested and valued features RES-Q offers, however it is also the feature we had the greatest difficulty implementing. RES-Q currently provides descriptive statistics, graphs, and customizable presentations for national and hospital level data on an annual basis. This must be improved to meet the needs of collaborators

Quarterly reporting. The aforementioned reports will be generated and provided to national coordinators

Real-time reporting web application. This will be in addition to the standard reporting functionality. A webaccessible dashboard will allow users to see their current performance in key metrics such as enrolment, door-to-needle, door-to-groin, and recanalization rate.

\section{Additional data entry options}

Another key functional area that we identified as deficient was the requirement for computer access to enter RES-Q data. As RES-Q is targeted at resolving treatment disparities related to resource availability, it is important that even stroke treatment centres with limited computing resources should be able to monitor their treatment performance. To accommodate this need, we have already collaborated with the Angels Initiative to allow for data entry to RES-Q from the Angels Initiative Stopwatch App, as even in areas where computers are limited, mobile phones are still frequently available.

In addition to mobile app data entry, we also intend to implement data entry via paper forms using OCR technology. This will allow centres to collect the same metrics, using the same questionnaire as every other RESQ participating site with only a pencil and paper. 